\title{
Fuzzy Clustering Algorithms for Effective Medical Image Segmentation
}

\author{
Deepali Aneja \\ Department of Electronics and Communication Engineering, Netaji Subas Institute of Technology, New Delhi, India \\ E-mail: deepalianeja@gmail.com \\ Tarun Kumar Rawat \\ Department of Electronics and Communication Engineering, Netaji Subas Institute of Technology, New Delhi, India \\ E-mail:tarun@nsit.in
}

\begin{abstract}
Medical image segmentation demands a segmentation algorith $m$ which works against noise. The most popular algorith $\mathrm{m}$ used in image segmentation is Fuzzy C-Means clustering. It uses only intensity values for clustering which makes it highly sensitive to noise. The comparison of the three fundamental image segmentation methods based on fuzzy logic namely Fuzzy C-Means (FCM), Intuitionistic Fuzzy C-Means (IFCM), and Type-II Fuzzy C-Means (T2FCM) is presented in this paper. These algorith $\mathrm{ms}$ are executed in two scenarios- both in the absence and in the presence of noise and on two kinds of images-Bacteria and CT scan brain image. In the bacteria image, clustering differentiates the bacteria from the background and in the brain CT scan image, clustering is used to identify the abnormality region. Performance is analyzed on the basis cluster validity functions, execution time and convergence rate. Misclassification error is also calculated for brain image analysis.
\end{abstract}

Index Term - Fuzzy Clustering, Fu zzy C-Means, FCM Type-II, Intuitionistic FCM, Fuzzy Set

\section{Introduction}

In computer vision, image segmentation is one of the most stimulating and difficult problems in the image processing which is used in a variety of applications such as machine vision, object recognition, and medical imaging [1 - 3]. Image segmentation helps in dividing of an image into multiple segments which makes it easier to analyze and understand. As a result, a set of separate regions can be achieved with even and homogeneous features such as texture, intensity, tone, color etc. Any particular segmentation technique is not defined for all images. Mostly all the segmentation techniques aim at any given concrete problem as there is not a universal segmentation method. Unlike Hard clustering, in fuzzy clustering (or soft clustering), data elements can fit to more than one cluster, and membership level is linked with each element.
Bezdek (1981) [4], proposed Fuzzy c-means algorithm (FCM), and it has been extensively used in the image segmentation $[5,6]$. The belongingness of each image pixel is never crisply defined and hence the introduction fuzziness makes it possible for the clustering techniques to preserve more information. It is known that an image can be characterized in various feature spaces. Data points are combined to form individual clusters in the feature domain. This is the fundamental princip le for Fu zzy C-Means functionality. The associated cost function is iteratively minimized, and the distance of pixels to the cluster centers in the feature domain is used to calculate the cost function. Incorrect FCM clustering results are obtained in case the image is corrupted with noise because of its anomalous feature data. Many researchers have come up with a different kind of approaches to compensate this shortcoming of FCM.

Modification to the FCM algorith m was proposed by Rhee and Hwang [7] which resulted in Type-II fuzzy clustering (T2FCM). Fuzziness in a fuzzy set defines the Type-II fuzzy set. The membership value of each arrangement in the image is extended by assigning Type-II fuzzy membership to conventional FCM. The cluster center equation is updated according to the new Type -II fuzzy membership. T. Chaira [8] utilized the intuitionistic fuzzy set theory and proposed the intuitionistic fuzzy c-means algorith $\mathrm{m}$. In this algorithm, a new uncertainty factor in incorporated in the membership function called as the hesitation degree that is incorporated. The structure of this paper is given as: Section II presents the review of the three clustering algorithms namely Fuzzy C-Means (FCM), Type-2 FCM (T2FCM) and Intuitionistic Fuzzy C-means (IFCM). Qualitative and Quantitative analysis of the performance is presented for the bacteria image and the brain CT scan image in section III. The results are concluded in the last section of the paper i.e. section IV. 


\section{Review of Algorithms}

The image is converted into a data-set of pixels which is denoted by ' $\mathrm{Y}$ ', where $\mathrm{Y}=\left\{\mathrm{y}_{1}, \mathrm{y}_{2}, \mathrm{y}_{3} \ldots \mathrm{y}_{\mathrm{n}}\right\}$. This specifies that an image with pixels in $\mathrm{N}$ dimensional space has to be partitioned into ' $r$ ' clusters. These algorithms are based on the distance $\left(d_{i p}\right)$ between the centroid of the cluster $\left(\mathrm{v}_{\mathrm{i}}\right)$ and the particular pixel $\left(y_{p}\right)$.

\subsection{The Fuzzy C-Means Algorithm}

FCM [4] is the standard fuzzy clustering algorithm. The assumption made in this technique is the prior knowledge of the number of clusters ' $r$ '. The distance $\mathrm{d}_{\mathrm{ip}}=\left\|\mathrm{y}_{\mathrm{p}}-\mathrm{v}_{\mathrm{i}}\right\|$ represents how far is pixel $\mathrm{y}_{\mathrm{p}}$ from the cluster center $v_{i}$. The membership of pixel ' $y_{p}$ ' in the ' $i{ }^{\text {th, }}$ cluster is represented as $u_{i p}$. It is defined as:

$$
\sum_{i=1}^{r} u_{i p}=1 ; p=1,2, \ldots . n
$$

The membership function gives the probability that a pixel belongs to a specific cluster. In this algorith $\mathrm{m}$, the probability is based solely on the distance between the individual cluster center and the pixel in the feature domain. When the location of a new cluster center is updated, the degree of membership depicts how much each pattern contributes in adjusting the new cluster center location. A constant ' $q$ ' is defined as the controlling factor for the fuzziness of the resulting partition. It is also referred as the fuzzifier.

The membership function and cluster centers are updated as:

$$
u_{i p}=\frac{1}{\sum_{j=1}^{r}\left(\frac{d_{i p}}{d_{j p}}\right)^{\frac{2}{q-1}}} \forall p, i
$$

where $1 \leq \mathrm{i} \leq \mathrm{r} ; 1 \leq \mathrm{p} \leq \mathrm{n}$

and

$$
v_{i}=\frac{\sum_{p=1}^{n}\left(u_{i p}^{q} y_{p}\right)}{\sum_{p=1}^{n}\left(u_{i p}^{q}\right)} \forall i
$$

The goal is to minimize the objective function $\left(\mathrm{J}_{\mathrm{FCM}}\right)$ as follows:

$$
J_{F C M}=\sum_{i=1}^{r} \sum_{p=1}^{n} u_{i p}^{q} d_{i p}^{2}
$$

Generally used feature used in image clustering is the gray-level value or the intensity of the pixel. The assignment of the membership values depends on the distance between the pixel point and the cluster centroid. To minimize the cost function for FCM, low membership values are assigned when the point is far from the centroid and vice versa. In order to achieve that, $\mathrm{J}_{\mathrm{FCM}}(\mathrm{U}, \mathrm{V})$ is iteratively updated with the continuous update of the membership function and the cluster center, until $\left|\mathrm{U}^{(\mathrm{z}+1)}-\mathrm{U}^{(\mathrm{z})}\right|<=\beta$, where ' $\mathrm{z}$ ' represents the number of iterations.

FCM works well for the noiseless images, but if the image is distorted or noisy then it misclassifies noisy pixels. This drawback of misclassification is the pixel intensity based calculation.

\subsection{The Type-2 Fuzzy C-Means (T2FCM)}

The next approach after FCM was to focus on the estimation of cluster centers to converge to a more desirable location even in the presence of noise. Rhee and Hwang [7] extended the straight FCM membership values to Type-II FCM. In this algorithm, membership function is assigned to each me mbership value of FCM. A modified Type-II membership is derived as:

$$
a_{i p}=u_{i p}-\frac{1-u_{i p}}{2}
$$

where $a_{i p}$ is the Type-II and $u_{i p}$ is the Type-I fuzzy membership. The equation for the centroid of the cluster remained unchanged. Updating the cluster center is obtained by substituting (5) in (3) as shown:

$$
v_{i}=\frac{\sum_{p=1}^{n}\left(a_{i p}\right)^{q} y_{p}}{\sum_{p=1}^{n}\left(a_{i p}\right)^{q}}
$$

An improved typicality can be seen in the Type-II me mbership values because Type-II cluster centers tend to have more appropriate locations than the Type-I cluster centers in noise corrupted images. This happens due the decline in the contribution of the pattern of pixels to any given cluster that has low memberships. The algorith $m$ continues till a point where the previous membership and the updated membership agree as:

$$
\max _{i p}\left|a_{i p}^{n e w}-a_{i p}^{p r e v}\right|<\beta
$$

$\beta$ is a userdefined value.

Hence the only difference between the Type-II and Type-I is given by (6). Similar to FCM, the cost function has to be minimized, and the cluster center is updated at every iteration. Type-II FCM has been effective for data sets like diamond and square, but when the application comes to complex patterns and images, it fails. 


\subsection{Intuitionistic Fuzzy C-Means (IFCM)}

In case of digital images, it can't be accurately defined which pixel belongs to exactly which cluster. There is some kind of hesitation related to the definition of the membership function. This idea lead to the idea of the higher fuzzy set by Atanassov in 1983 [8] called as intuitionistic fuzzy set.

IFCM [9] objective function is derived from two basic terms: (i) Intuitionistic fuzzy set based objective function and (ii) new intuitionistic fuzzy entropy (IFE). The new IFCM objective function is defined as:

$$
J_{I F C M}=\sum_{i=1}^{r} \sum_{p=1}^{n} u_{i p}^{q} d_{i p}^{2}+\sum_{i=1}^{r} \pi_{i}^{*} e^{1-\pi_{i}^{*}}
$$

The advanced intuitionistic membership function has an additional component to incorporation the indecisiveness. It is defined as:

$u_{i p}^{*}=u_{i p}+\pi_{i p}$, where $u_{i p}^{*}$ denotes the intuitionistic fuzzy and $u_{i p}$ is the conventional FCM membership function. It denotes the probability of the $\mathrm{p}^{\text {th }}$ data in the $\mathrm{i}^{\text {th }}$ class.

$\pi_{i p}$ is hesitation factor, which is given as:

$$
\pi_{i p}=1-u_{i p}-\left(1-u_{i p}^{\alpha}\right)^{1 / \alpha}
$$

and

$$
\pi_{i}^{*}=\frac{1}{N} \sum_{p=1}^{n} \pi_{i p,} \quad p \in[1, N]
$$

Intuitionistic fuzzy entropy (IFE) constitutes the second term in the objective function. Its addition is done to make sure the maximization the good points in the class and minimizing the entropy of the histogram of an image is the goal.

Cluster centers are modified as:

$$
v_{i}=\frac{\sum_{p=1}^{n} u_{i p}^{*} y_{p}}{\sum_{p=1}^{n} u_{i p}^{*}}
$$

The membership matrix and the cluster center are updated with every iteration. The algorith $m$ terminates when the updated membership and the previous membership agree to the following condition:

$$
\text { i.e. } \max _{i p}\left|U_{i p}^{* \text { new }}-U_{i p}^{* \text { prev }}\right|<\beta \text {, }
$$

$\beta$ is a userdefined value.

\section{Simulations \& Results}

Simulation is done using MATLAB v. 7.0 on the computer with the specifications - Intel ${ }$ Core $^{\mathrm{TM}}$ 2DUO CPU T7250@2.00 GHz and 3.45GB of RAM. We have assumed the value of $\beta=0.002, \alpha=0.85, q=2$ according to the most common choice for clustering. Also, assumption is made for the total number of iterations as 200 .

Two kinds of analysis is performed - Qualitative and quantitative and shown for both the images. The performance analysis parameters are chosen as in [10].

\subsection{Bacteria Image}

The specification for the bacteria image considered for the experimentation is $120 \times 142 \times 3$ pixels. The goal of the algorithm is to separate the bacteria from the background efficiently. Implementation of all three algorith $\mathrm{ms}$ is done on both kinds of images - noiseless and corrupted with noise. Gaussian noise is introduced with $3 \%$ intensity and the image consists of two clusters. Various types of noises and levels of noise percentages have been experimented with images to show the performance of all the clustering algorith ms [11]. Fig. 1(a) represents the original noiseless bacteria image, and the clustering outcome is shown in Fig 1(b)-(d) for three clustering methods FCM, T2FCM and IFCM respectively. Fig 2(a) represents the noise corrupted bacteria image and Fig 2(b)-(d) represent results on the noisy image for three algorithms.

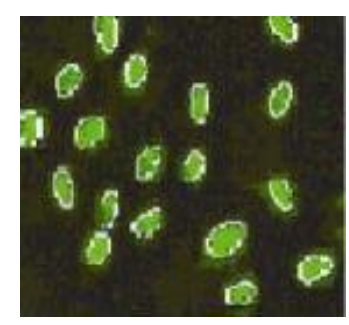

(a)

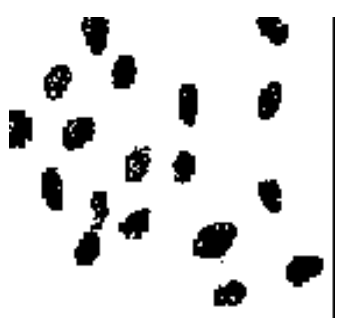

(b)

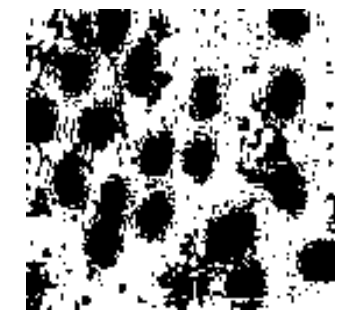

(c)

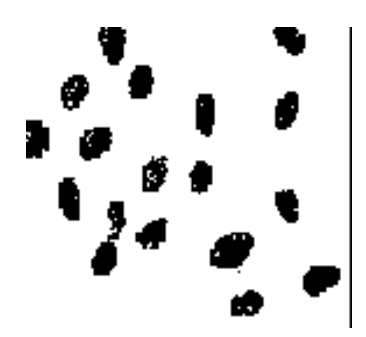

(d)

Fig. 1: (a) Bacteria original image, (b) FCM cluster, (c) Type-II FCM cluster, and (d) IFCM cluster 


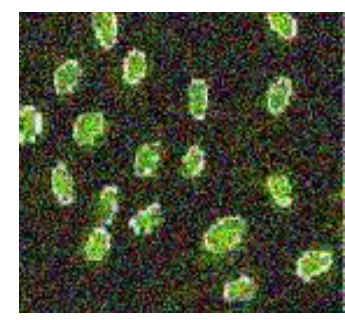

(a)

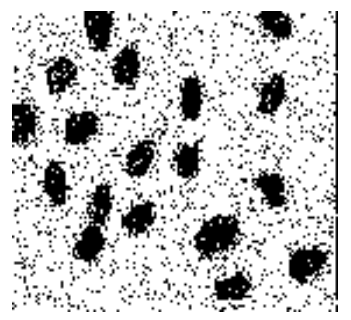

(b)

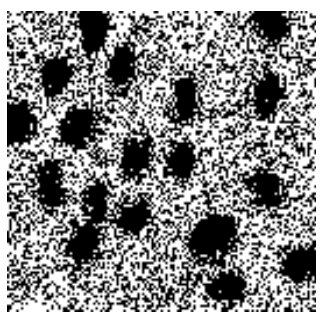

(c)

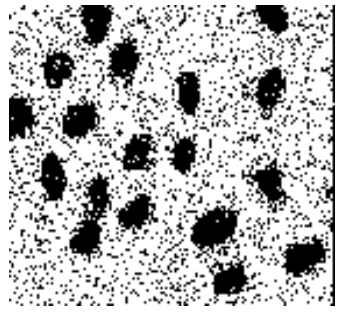

(d)

Fig. 2: (a) Bacteria noisy image, (b) FCM cluster, (c) Type-II FCM cluster, and (d) IFCM cluster

Noiseless image results depict the best performance for FCM algorithm and IFCM algorithms. T2FCM produces an image with blurred boundaries and augmented size of bacteria. In case of noise corrupted image, IFCM tops the result with removal of noise as well as retaining the boundaries of bacteria. T2FCM does better as compared to FCM in removal of noise, but at the cost of increased bacteria size.

\subsection{CT Scan Brain Clot Image}

A Brain CT scan image $(242 \times 248 \times 3$ pixels $)$ is considered with a hemorrhage/ clot as an application for fuzzy c-means clustering techniques. The image consists of four clusters, and our aim is to consider the cluster distinguishing the clot from the background. Analysis is done by simulating the algorithms on the poorly illuminated CT scan clot image represented in Fig 3(b). For comparis on of the outcome, the ground truth image represented in Fig 3(a) is considered. From medical point of view, various components of the image are shown as the lateral ventricles, third ventricle and a blood clot (or a hemorrhage region). In case of Brain CT scan image, it can be shown and validated [8] that with $\alpha \leq 0.5$, the resultant images are not properly clustered. Performing the experiment at $\alpha=0.6$ (i.e. $\alpha>$
0.5 ), we get a binary thresholded image is obtained and with $\alpha>0.5$, i.e. clustered images are obtained, but better results are obtained for increased value of $\alpha=0.7$. After experimenting with various values of $\alpha$, to obtain the best result, $\alpha=0.85$ is used. The clustering results on the experimental image are shown in Fig 3(c)-(e) for FCM, T2FCM and IFCM respectively. The aim is to detect the clot region with the matching size as given in the ground truth image. IFCM shows the best result with almost no noise and clear detection of the clot region. The area of the clot size is almost equal as that in the ground truth image. In case of FCM, the clot is detected, but with the reduced size. On the contrary, T2FCM identifies the clot with an increase in the clot size. The gray matter is noisier in FCM outcome than Type II FCM. IFCM not only shows a better detection, it also identifies the other regions clearly.

Hence, in the absence of noise, FCM stands first along with IFCM producing equivalent results. In case of noise corrupted image, IFCM shows the best clusters including both the parameters - size of the cluster and the removal of the noise. FCM fails in the presence of noise, whereas T2FCM gives unpredictable and erratic results with varying the particular region and the kind of image.

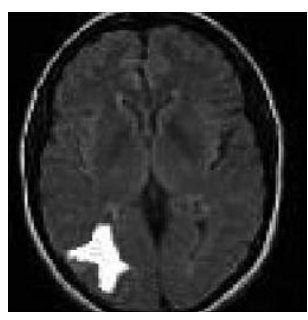

(a)

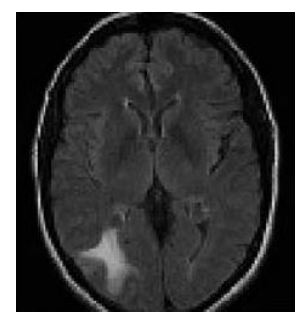

(b)

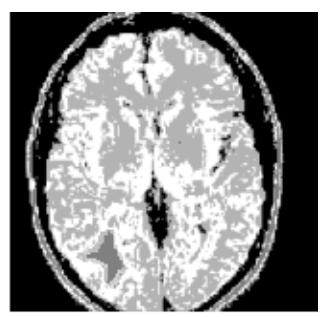

(c)

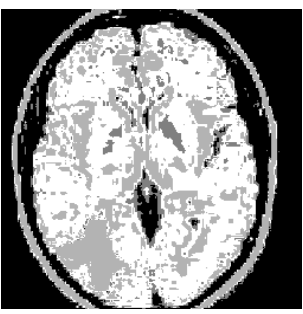

(d)

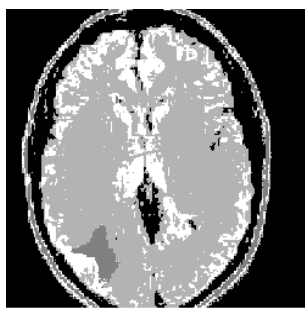

(e)

Fig. 3: (a) Ground truth image, (b) CT scan clot image, (c) FCM cluster, (d) T2FCM cluster, and (e) IFCM cluster

\subsection{Various parameters for performance}

\section{(i) Cluster Validity Functions}

In order to analyze the rationality of the result obtained after clustering, cluster validity functions are used. After the visual analysis in the previous section, we also calculated quantitative impact of clustering by using various cluster validity functions, to measure the accuracy. 
Most commonly used valid ity functions are based on the fuzzy partition of the data set as they are simple and easy to implement. Out this group, we used two functions namely the feature structure and the fuzzy partition. The fuzzy partition comprises of two components namely partition coefficient [12] and partition entropy [13]. They are represented in (11) and (12). Partition coefficient should be greater and partition entropy should be lesser for the best clustering results.

$$
\begin{gathered}
V_{p c}(U)=\sum_{i=1}^{n} \sum_{p=1}^{r} u_{p i}^{2} \\
V_{p e}(U)=-\frac{1}{n}\left\{\sum_{i=1}^{n} \sum_{p=1}^{r}\left[u_{p i} \log u_{p i}\right]\right\}
\end{gathered}
$$

They do not incorporate the featuring attribute or property which is the main drawback with these two functions. To solve this issue, other feature based validity functions are used [14], [15]. They are Fukuyama-Sugeno and Xie-Beni shown in (13) and (14) respectively.
They are defined as:

$$
\begin{aligned}
V_{f S}(U, V ; Y)= & \sum_{p=1}^{r} \sum_{i=1}^{n} u_{i p}^{q}\left(\left\|y_{i}-v_{p}\right\|^{2}\right. \\
& \left.-\left\|v_{p}-\bar{v}\right\|^{2}\right)
\end{aligned}
$$

where $\bar{v}=\frac{1}{r} \sum_{p=1}^{r} v_{p}$

$V_{x b}(U)$

$$
=-\frac{\sum_{p=1}^{r} \sum_{i=1}^{n} u_{p i}^{q}|| y_{i}-v_{p}||^{2}}{n *\left(\min _{p \neq j}\left\{|| v_{p}-v_{j}||^{2}\right\}\right)}
$$

Either of $V_{f s}$ or $V_{x b}$ should be minimal, for good clustering results.

Performance comparison of FCM, T2FCM, and IFCM is shown in TABLE-I in terms of these four cluster validity functions.

Table I: Performance comparison of FCM, TYPE-II FCM, and IFCM in terms of Cluster Validity Functions

\begin{tabular}{|c|c|c|c|c|c|c|}
\hline \multirow{2}{*}{ Image } & \multirow{2}{*}{ No. of clusters } & \multirow{2}{*}{ Method } & \multicolumn{4}{|c|}{ The value of Validity functions } \\
\cline { 3 - 7 } & & & $\mathbf{V}_{\mathbf{f s}}$ & $\mathbf{V}_{\mathbf{p c}}$ & $\mathbf{V}_{\mathbf{p e}}$ & $\mathbf{V}_{\mathbf{x b}}$ \\
\hline \multirow{3}{*}{$\begin{array}{c}\text { Noisy } \\
\text { Bacteria image }\end{array}$} & 2 & $\mathrm{FCM}$ & 3804.9 & 0.71965 & 0.44027 & 0.23954 \\
\cline { 3 - 7 } & & $\mathrm{T} 2 \mathrm{FCM}$ & 1321.1 & 0.4243 & $-0.13601+1.8024 \mathrm{i}$ & 0.50455 \\
\cline { 3 - 7 } & $\mathrm{IFCM}$ & 2214.3 & 0.50007 & 0.6935 & 6636.2 \\
\hline \multirow{3}{*}{$\begin{array}{c}\text { CT scan Brain } \\
\text { Clot image }\end{array}$} & \multirow{2}{*}{4} & $\mathrm{FCM}$ & -23006 & 0.83285 & 0.30756 & 0.027236 \\
\cline { 3 - 7 } & & $\mathrm{T} 2 \mathrm{FCM}$ & 12473 & 2.3203 & $-0.48133+8.1794 \mathrm{i}$ & 15.853 \\
\cline { 3 - 7 } & $\mathrm{IFCM}$ & -21869 & 0.83316 & 0.30872 & 0.21713 \\
\hline
\end{tabular}

\section{(ii) Execution time}

TABLE II shows the outcome for FCM, T2FCM and IFCM in terms of the convergence rate and the execution time. FCM technique has least execution time compared to other image seg mentation techniques. It is can be seen that IFCM method takes much more time to execute, but has the best convergence rate as the number of iterations is the least in both images.

Table II: Performance comparison of FCM, TYPE-II FCM, and IFCM in terms of Execution time and Convergence rate

\begin{tabular}{|c|c|c|c|}
\hline Image & Method & Execution time (seconds) & No. of iterations (conv. Rate) \\
\hline \multirow{3}{*}{ Noisy Bacteria } & FCM & 0.361 & 30 \\
\cline { 2 - 4 } & T2FCM & 0.297 & 23 \\
\cline { 2 - 4 } & IFCM & 1.125 & 3 \\
\hline \multirow{3}{*}{ CT scan Brain Clot } & FCM & 3.906 & 46 \\
\cline { 2 - 4 } & T2FCM & 9.54 & 95 \\
\cline { 2 - 4 } & IFCM & 65.312 & 32 \\
\hline
\end{tabular}




\section{(iii) Misclassification error in brain image}

This parameter gives an idea about the misclassification of the resultant cluster being identified as compared to the ground truth image or manually segmented image. Ground image is manually segmented using FCM as it is already observed that it gives the best outcome in the absence of any disturbance or noise. For all the methods, the misclassification error is calculated. It is defined as [16]

$$
\text { Error }=\frac{\left|B_{E} \cap B_{G}\right|+\left|F_{E} \cap F_{G}\right|}{B_{G}+F_{G}}
$$

where $B_{G}$ and $B_{E}$ represent the background image pixels of the ground truth image and experimental image respectively. $F_{G}$ and $F_{E}$ are the foreground area pixels of the ground and experimental image respectively. The region other than the concerned (clot in this experiment) is considered as the background region.

In case of CT scan Brain image and is defined as:

$$
\text { Error }=\frac{\left|R_{E}^{C} \cap R_{G}^{C}\right|+\left|R_{E}^{B} \cap R_{G}^{B}\right|}{R_{G}^{C} T^{+} R_{G}^{B}}
$$

where $R_{E}^{C}$ represents the clot region of the experimental image and $R_{G}^{C}$ denote the same region for the ground truth image. $R_{G}^{B}$ and $R_{E}^{B}$ are the background regions of the ground truth image and the experimental image respectively.

TABLE-III shows the percentage of misclassification error in all the three algorithms. It can be observed that IFCM demonstrates the best performance with the least \% of misclassification error.

Table III: Percentage of Misclassification Error

\begin{tabular}{|c|c|c|c|}
\hline Image & FCM & T2FCM & IFCM \\
\hline CT scan Brain clot & 1.283 & 0.962 & 0.537 \\
\hline
\end{tabular}

\section{Conclusion}

Clustering focuses on finding the boundaries of the desired object with precision. Medical images generally contain some percentage of noise and a considerable level of uncertainty. Clustering techniques can also be used to identify any region of abnormality in the noisy experimental images.

This paper, presents a comparison between three fuzzy based techniques namely - Fuzzy C-Means (FCM), Intuitionistic Fuzzy C-Means (IFCM), and Type-II Fuzzy C-Means (T2FCM). Experiments have been performed for two different kinds of images Bacteria and CT scan Brain image with a hemorrhage/ clot region. Two different types of conclusions are obtained on inspecting the results.

For noiseless images, Fuzzy C-Means algorithm produced the best results, with IFCM finishing as a close contender and Type-II FCM is nowhere close. In the presence of noise, T2FCM is better than FCM, whereas IFCM out rightly stands at the top with the best segmentation results in comparison to the other two algorithms. T2FCM did a good job in removing noise at the cost of relative increase in size of the bacteria, whereas FCM does nothing to remove the noise in the bacteria. In case of the CT scan brain image, T2FCM algorithm, amplified the size of the clot and FCM reduced the size of the clot. So, we can conclude that T2FCM does not have the characteristics of an efficient technique for image segmentation. It may have had presented good theoretical results, but the practical results are not in agreement with those obtained in theory. Also, IFCM takes the least no. of iterations and gives the least percentage of misclassification error. Experiments can be performed with some other algorithms on the same images to get better results.

\section{References}

[1] J. C. Bezdek, L. O. Hall, L. P. Clarke. Review of MR image segmentation techniques using pattem recognition. Medical Physical, 1993, 20(4): 10331048.

[2] D. L. Pham, C. Y. Xu, J. L. Prince. A survey of current methods in medical image segmentation. Annual Review of BiomediclEngineer, 2000, 2: 315-337.

[3] W. M. Wells, W. E. Lgrimson, R. Kikinis, et al. Adaptive segmentation of MRI data. IEEE Trans. on Medical Imaging, 1996, 15(4): 429-442.

[4] J. C. Bezdek. Pattern recognition with fuzzy objective function algorithms. New York: Plenum Press, 1981.

[5] J. K. Udupa, S. Samarasekera. Fuzzy connectedness and object definition: theory, algorithm and applications in image segmentation.Graphical Models Image Processing, 1996, 58(3):246-261.

[6] S. M. Yamany, A. A. Farag, S. Hsu. A fuzzy hyperspectral classifier for automatic target recognition (ATR) systems. Pattern Recognition Letters, 1999, 20: 1431-1438.

[7] F.C.H. Rhee, C. Hwang, A Type-2 fuzzy c means clustering algorithm, in: Proc. in Joint 9th IFSA World Congress and 20th NAFIPS International Conference 4, 2001, pp. 1926-1929.

[8] Atanassov's, Intuitionistic fuzzy sets, VII ITKR's Session, Sofia, 983 : Deposed in Central Science - 
Technology Library of Bulgaria Academy of Science - 1697/84.

[9] T. Chaira, "A novel intuitionistic fuzzy c means clustering algorith $\mathrm{m}$ and its application to medical images", Applied Soft computing 11(2011) 1711 1717.K.T.

[10] P.Kaur, P.Gupta, P.Sharma (2012), “Review and comparis on of kernel based image segmentation techniques", IJISA, 2012, 7, 50-60

[11] P.Kaur, N.Chhabra (2012), "Image Segmentation Techniques for Noisy Digital Images based upon Fuzzy Logic- A Review and Comparison", IJISA 2012, 7, 30-36.

[12] Bezdek JC.(1974), "Cluster validity with fuzzy sets”, J Cybern 1974; 3:58-73.

[13] Bezdek JC.(1975), "Mathematical models for systematic and taxonomy", In: proceedings of eigth international conference on numerical taxonomy, San Francisco; 1975, p. 143-66.

[14] Fukuyama Y, Sugeno M. (1989), “A new method of choosing the number of clusters for the fuzzy cmeans method", In: proceedings of fifth fuzzy systemsymposium; 1989, p. 247-50.

[15] Xie XL, Beni GA. (1991), "Validity measure for fuzzy clustering”, IEEE Trans Pattern Anal Mach Intell 1991;3:841-6.

[16] W.A. Yasnoff, et al., Error measures for scene segmentation, Pattern Recognition 9 (1977) 217231.

\section{Authors' Profile}

Deepali Aneja completed her B. Tech. in 2008. She is pursuing her Masters of Technology in Signal Processing (ECE) from Netaji Subas Institute of Technology, New Delhi. Currently, she is working on her Masters Dissertation and this paper is the implementation of her thesis work. Her research interests are Image Seg mentation and Signal Processing.

Tarun Kumar Rawat is currently Lecturer, Department of Electronics and Communication Engineering, Netaji Subhas Institute of Technology (NSIT), Delhi. His teaching and research interests are in the areas of Circuits \& Systems, Digital Signal Processing, Statistical Signal Processing, Stochastic Nonlinear Filters, and Digital Communication.

How to cite this paper: Deepali Aneja, Tarun Kumar Rawat,"Fuzzy Clustering Algorithms for Effective Medical Image Segmentation", International Journal of Intelligent
Systems and Applications(IJISA), vol.5, no.11, pp.55-61, 2013. DOI: $10.5815 /$ ijisa.2013.11.06 